ICU. Nonalcoholic WE should be considered as a possible diagnosis when the classic triad is absent. Prognosis of the patient mostly depends on early recognition of the syndrome and early initiation of thiamine treatment. It may be fatal if it progresses to Korsakoff syndrome. Thus, higher suspicion and recognition of WE in early stage can increase chances of full reversal of this condition.

\section{A0027 Patient with Hurler's Syndrome for Emergency Ventriculoperitoneal Shunt: What an Anesthesiologist Must Know \\ Teena Bansal, ${ }^{1}$ Shashi Kiran, ${ }^{1}$ Savita Saini, ${ }^{1}$ Tarun Yadav ${ }^{1}$ ${ }^{1}$ Department of Anaesthesiology and Critical Care, Pt. B. D. Sharma Post Graduate Institute of Medical Sciences, Rohtak, Haryana, India}

Background: Patients with Hurler's syndrome pose a great challenge to anesthesiologists. The most common problem faced by anesthesiologist is difficult venous access and establishment and maintenance of difficult airway.

Case Description: A 22-year-old female patient, a known case of Hurler's syndrome, presented with complaint of headache. She was scheduled for insertion of VP shunt for acute hydrocephalus. History was significant for obstructive sleep apnea, and she used CPAP during sleep for the same. The patient was cooperative but slow in response to commands. General physical examination revealed short stature, dysmorphic face, macrocephaly, frontal bossing, short neck, depressed nasal bridge, dorsolumbar kyphosis, flexion deformity of elbows and wrists, and stubby fingers. Airway evaluation revealed large tongue and Mallampati grade IV. Echocardiography revealed mitral valve prolapse with trivial mitral regurgitation. Intravenous access was obtained with difficulty. Difficult mask ventilation was anticipated, in addition to difficult laryngoscopy and intubation. Awake fiberoptic intubation was performed successfully. Anesthesia was induced, and at the end of surgery, trachea was extubated awake.

Conclusions: The most important step in management of airway is to avoid a situation of "can't ventilate, can't intubate." The basic aim is preservation of spontaneous ventilation and is of utmost importance. Recovery after general anesthesia is often slow in these patients and may be accompanied by breath-holding, apnea, and bronchospasm. The patient should be awake with adequate airway reflexes for extubation.

\section{A0028 Effect of Comorbidities on the Outcome of Patients with Aneurysmal Subarachnoid Hemorrhage: A Prospective Observational Study \\ Sunaakshi Puri, ${ }^{1}$ Hemant Bhagat, ${ }^{1}$ Kiran Jangra \\ ${ }^{1}$ Department of Anaesthesia and Intensive Care, Postgraduate Institute of Medical Education and Research, Chandigarh, India}

Introduction: Subarachnoid hemorrhage (SAH) carries a high mortality of 30 to $40 \%$. Amongst survivors, 40 to $50 \%$ suffer disability and cognitive decline. Comorbidities can have a contribution in the disease process. We hypothesize that the presence of comorbidities in a patient with aneurysmal SAH can influence the course of disease and thereby the neurological outcome.

Materials and Methods: This prospective observational study was commenced after ethical committee clearance and written informed consent. We enrolled 140 (94 females and 46 males) consecutive patients with aneurysmal SAH, scheduled for clipping/coiling from July 2016 to October 2017. Patients' comorbidities were recorded and outcome followed in postoperative period using MRS at discharge and mRS and GOS at 1 month after discharge. Analysis was done using chi-square test.

Results: In patients undergoing clipping, HTN and smoking are associated with worse outcome (0.02 and 0.01 , respectively). HTN is associated with increased incidence of vasospasm and infarct while smoking and CAD are associated with increased incidence of rebleed and hydrocephalus, respectively. In patients undergoing coiling, HTN, smoking, and CAD are associated with worse outcome $(0.02,0.04$, and 0.02 , respectively). Smoking, alcohol intake, and CAD are associated with increased incidence of rebleed while CAD is associated with increased incidence of hydrocephalus. DM, thyroid dysfunction, TB, and joint disease are not associated with worse outcome or increased incidence of complications.

Conclusions: Various comorbidities including HTN, CAD, smoking, and alcohol intake were associated with increased risk of perioperative complications in patients after $\mathrm{SAH}$. Other comorbidities such as DM, thyroid dysfunction, $\mathrm{TB}$, and joint disease neither affect outcome nor increase risk of complications.

A0029 Measurement of Neutrophil GelatinaseAssociated Lipocalin for Acute Kidney Injury following Neuroradiological Procedure(s) in Patients with Aneurysmal Subarachnoid Hemorrhage: A Preliminary Study

Ashutosh Kaushal, ${ }^{1}$ Ashish Bindra, ${ }^{1}$ Rajendra S. Chouhan ${ }^{1}$ ${ }^{1}$ Department of Neuroanaesthesiology and Critical Care, All India Institute of Medical Sciences, New Delhi, India

Background: Patients with aneurysmal SAH are susceptible to acute kidney injury (AKI) due to preexisting hypovolemia, renal hemodynamic changes, and neuroendocrine response. NGAL represents a promising biomarker for early prediction, monitoring treatment response, and prognostication of AKI in various clinical settings. The purpose of this study was to find incidence of AKI and to see changes in plasma NGAL at different time periods in aneurysmal SAH patients undergoing neuroradiological intervention.

Materials and Methods: All consenting aneurysmal SAH patients (18-65 years, modified Hunt \& Hess grade 1-4) posted for neuroradiological procedures during the study time were included. Patients with history of chronic renal disease, recent contrast exposure, and renal insufficiency due to any other reasons were excluded. Blood sample for serum creatinine and serum NGAL were obtained preprocedure and then at 1, 6, 24, and 48 hours after contrast administration. AKI was defined by KDIGO clinical practice guidelines. 